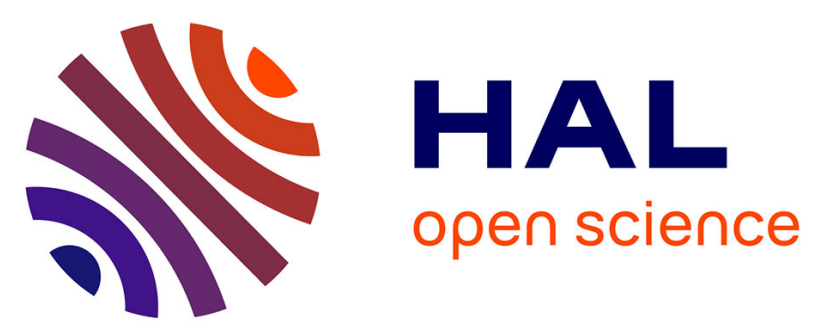

\title{
Low Cost Artificial Ventilator Embedding Unsupervised Learning for Hardware Failure Detection
}

Sebastián Marzetti, Pierre-Alexandre Peyronnet, Florent Barthélemy, Valentin

Gies, Valentin Barchasz, Thomas Delaey-Langlois, Daniel Peloux, Philippe

Arlotto, Hervé Barthélemy

\section{To cite this version:}

Sebastián Marzetti, Pierre-Alexandre Peyronnet, Florent Barthélemy, Valentin Gies, Valentin Barchasz, et al.. Low Cost Artificial Ventilator Embedding Unsupervised Learning for Hardware Failure Detection. IEEE Circuits and Systems Magazine -New Series-, 2021, 21 (3), pp.73-79. 10.1109/MCAS.2021.3092539 . hal-03517761

\author{
HAL Id: hal-03517761 \\ https://hal.science/hal-03517761
}

Submitted on 7 Jan 2022

HAL is a multi-disciplinary open access archive for the deposit and dissemination of scientific research documents, whether they are published or not. The documents may come from teaching and research institutions in France or abroad, or from public or private research centers.
L'archive ouverte pluridisciplinaire HAL, est destinée au dépôt et à la diffusion de documents scientifiques de niveau recherche, publiés ou non, émanant des établissements d'enseignement et de recherche français ou étrangers, des laboratoires publics ou privés. 


\title{
Low Cost Artificial Ventilator Embedding Unsupervised Learning for Hardware Failure Detection
}

\author{
Team : Sebastián Marzetti ${ }^{1}$, Pierre-Alexandre Peyronnet ${ }^{2}$, Florent Barthélemy ${ }^{2}$ \\ Mentors : Valentin Gies ${ }^{12}$, Valentin Barchasz ${ }^{1}$, Thomas Delaey-Langlois ${ }^{3}$, Daniel Peloux ${ }^{2}$, \\ Philippe Arlotto ${ }^{2}$, Hervé Barthélemy ${ }^{1}$ \\ ${ }^{1}$ Université de Toulon, Aix Marseille Univ, CNRS, IM2NP, Toulon, France \\ ${ }^{2}$ Université de Toulon, IUT de Toulon, France \\ ${ }^{3}$ Direction Générale de l'Armement (DGA), France
}

\begin{abstract}
In this paper, a less than $\$ 200$ artificial ventilator that can be used against COVID-19 pandemic is presented. Using low-cost easy-to-find materials, it has been designed for helping developing countries where supplies for building new medical equipment are limited. It complies with medical requirements, allowing to monitor and adjust ventilation parameters such as tidal volume, maximum intra-lung pressure and breath rate.

Even if this ventilator is low cost, focus has been placed on improving its global reliability. Using low-cost recycled materials may lead to mechanical failures, this potential drawback is addressed with an intelligent embedded hardware failure detector implemented inside the microcontroller. Using K-means optimized algorithm, it learns in a short time normal operation corresponding to the couple formed by a given ventilator set-up and a patient. In case of a mechanical breakdown, an alert is generated to inform medical staff.

First, mechanical, electrical and software architectures of the system are presented, then hardware failure detection algorithm is detailed. Finally, test results done at IRBA using an artificial lung are discussed. The overall project has been published as an open source one on GitHub: https://github.com/iutgeiitoulon/ArtificialVentilator.
\end{abstract}

Index Terms-Open source, Artificial Ventilator, Breathing Machine, Unsupervised Learning, K-Means, Machine Learning, Embedded Learning, Embedded system

\section{INTRODUCTION}

During COVID-19 initial lockdown period, a low cost (less than $\$ 200$ ) artificial ventilator has been designed at Toulon University, in France. It implements fully autonomous ventilation mode for reanimation, and assisted ventilation mode where patient breath start is detected and assisted by the ventilator. It complies with artificial ventilator medical requirements: limitation of inspired tidal volume, intra-lung pressure and adjustable breath rate [1] -[3].

Using easy-to-find low-cost materials such as fishing wire, aquarium tubing, hose and 3D printer stepper motors, this artificial ventilator can be built anywhere in the world through standard tools such as a jigsaw or a laser cutter. This makes it useful in emergency situations where it is not possible to have an access to a medically certified ventilator, such as during COVID-19 pandemic. However, quality of these materials is unknown. Therefore, in order to improve robustness, a

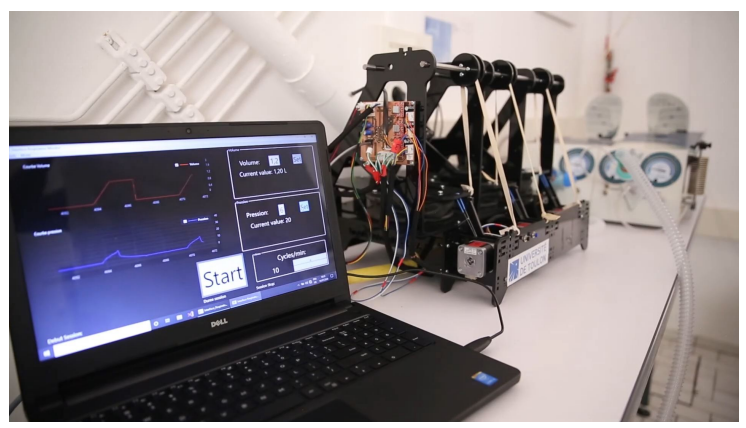

Fig. 1: Artificial ventilator being tested with an artificial lung

solution based on cooperation between embedded artificial intelligence and medical staff has been developed. It aims at warning medical staff when hardware failures are detected using unsupervised learning algorithms.

In order to help people, Toulon University students and researchers have decided to open source this project. Electronics, mechanics as well as embedded and monitoring software have been shared on GitHub: https://github.com/iutgeiitoulon/ArtificialVentilator. They can be used or modified freely.

The article is divided in five parts. Sections III and IV present ventilator mechanics, electronics and embedded software architecture. Section $\mathrm{V}$ presents monitoring software structure. Then section VI focuses on how to improve system robustness with an embedded failure detector using K-Means optimized algorithm. Finally, in section VII] results are presented and discussed.

\section{DISCLAIMER}

This artificial ventilator is not intended to become a commercial product used in a modern hospital. It has not been certified as compliant to state of art medical regulations and will not be asked for. However, it has been functionally tested successfully on an artificial lung as shown on Fig. 11 at IRBA (Institut de Recherche Biomédicale des Armées) 
in HIA (Hopital Inter-Armées) Sainte-Anne, Toulon, France. This test has been detailed in written report. However, Toulon University students and researchers cannot be liable of any damage, injury or death resulting from the use of this device.

\section{MechanicAl Architecture}

Proposed system mechanical architecture is presented in Fig. 2. It can be built using only material plates such as wood or plastic, using a jigsaw or a laser cutter.

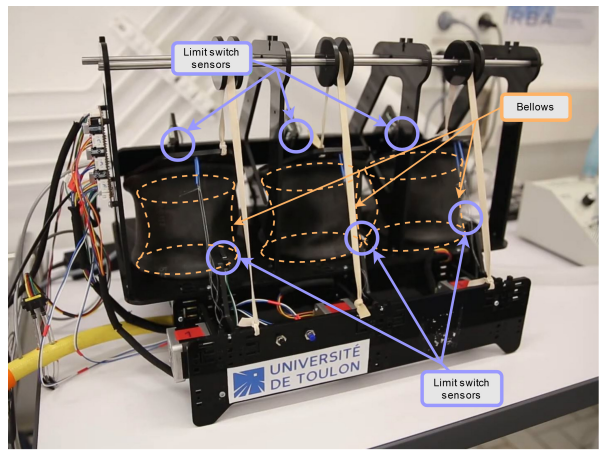

Fig. 2: Mechanical architecture of the artificial ventilator.

Mechanical structure is based on three bellows made from inner tire tube replacing the AMBU bag that is often used in artificial ventilators [4]-[7]. Apart from being easier to supply, using home-made bellows instead of AMBU bags add reproducibility to the system, since AMBU bags are quite different from each other, depending on the local provider. These differences have a highly negative impact on the maximum tidal volume capacity, which undermines the control system. Each bellow uses two limits sensors as shown in Fig. 2 and is operated by a three $3 \mathrm{D}$ printer stepper motor using fishing wire. Office rubber bands are used for repositioning bellows in their maximum volume position. Air or gas is directed by garden hoses and watering couplers, allowing important cost reduction, as well as easy implementation.

\section{Electrical ARChitecture}

Proposed electrical architecture is presented in Fig. 3 . Supplied with a hand-held power tool battery delivering $18 \mathrm{~V}$ DC, electronic system control is based on a Microchip PIC24FJ64GB004 microcontroller. There are two main sensors in the design: Silicon Micro Structures SM6221 for monitoring intra-lung pressure, and Omron D6F-PH0505AD3 for monitoring inspired tidal volume using a home-made Pitot tube, both connected in I2C. Omron D6F-PH0505AD3 is a precise differential pressure sensor having a measurement range from -50 to $50 \mathrm{~Pa}$, allowing to build a Pitot tube as shown in Fig. 4 Measured differential pressure $\Delta P$ is converted into air velocity using Euler equation [8]. Using fluid velocity and knowing hose pipe section, inspired volume $D$ can be calculated as shown in Eq. 1, where $t_{0}$ is the start of compression, $t_{1}$ its end, $V_{\text {Air }}$ the air velocity and $S_{P i p e}$ the hose pipe section.

$$
D\left[m^{3}\right]=\int_{t_{0}}^{t_{1}} V_{\text {Air }} * S_{\text {Pipe }} d t=\int_{t_{0}}^{t_{1}} \sqrt{\frac{2 \Delta P}{\rho}} * S_{\text {Pipe }} d t
$$

Actuators are Sanyo Denki 103H5208-5240 3D printer stepper motors driven by a Allegro A4988 motor driver. A serial link has been implemented in order to monitor all parameters on a standard $\mathrm{PC}$, without adding a difficult to find or expensive LCD directly to the ventilator.

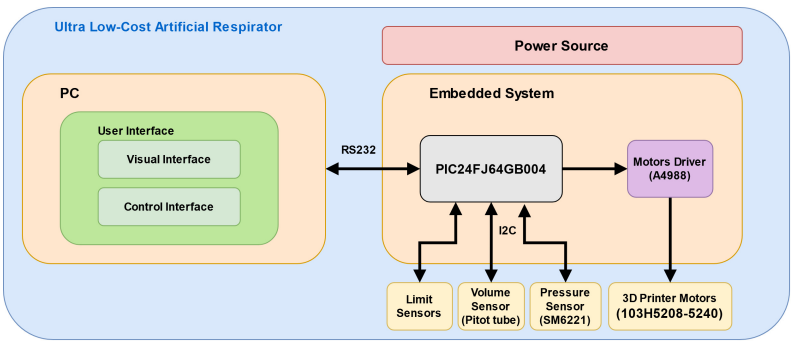

Fig. 3: Electronics block diagram.

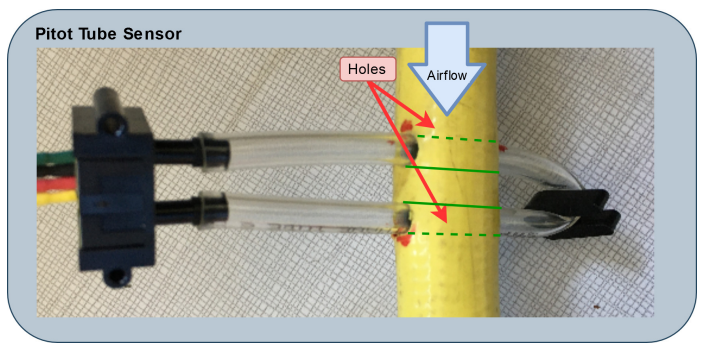

Fig. 4: Volume sensor implemented using home-made Pitot tube assembled on the Omron D6F-PH0505AD3 sensor.

\section{USER INTERFACE}

User graphic interfaces for supervision and set-up are developed in $C \#$, thus it can be run on a standard PC connected to the ventilator through a RS232 link. Sensors data is monitored in real time (intra-lung pressure and tidal volume). This interface allows the user to configure the following ventilator medical parameters:

- Inspired volume: can be set-up between 0.2 and $1.5 \mathrm{~L}$.

- Maximum intra-lung pressure (in millibars).

- Breath cycles per minute.

Interface also allows to set ventilator active mode among the following two ones:

- Reanimation: breath is initiated by the ventilator (usually used when patient is unconscious)

- Assistance: breath is initiated by the patient (patient is conscious and needs only an assistance)

It is important to note that computer interface is only used for monitoring and set-up. Artificial ventilator is fully controlled by its microcontroller, allowing an autonomous and reliable behaviour, even if computer link is lost for any reason. The full interface program is open source and published in [9].

\section{HARDWARE FAILURE AUTOMATIC DETECTION}

As low-cost recycled materials quality is unknown, a mechanical breakdown may occur. Therefore, in order to improve robustness, a solution based on cooperation between embedded artificial intelligence (AI) and medical staff is proposed. 
Embedded AI algorithm is based on characterizing interactions between the patient and the ventilator. This is done by extracting features from sensors signals as described in Section VI-A during breathing cycles, in order to later detect hardware failures or anomalies. It uses in situ unsupervised learning: every patient is different, so that it is not possible to learn system behaviour once and for all. Consequently, training process will be done every time the ventilator is used, under supervision of medical staff for a short time (about one minute), to check its proper functioning during that period.

Life of the patient cannot depend on the serial link between embedded microcontroller and computer interface. Thus, failure detection is fully implemented on the embedded microcontroller, so that a problem with $R S 232$ communication will not affect ventilator function, nor anomalies detection. Computer user interface software is used only for supervision and control. Hardware failure automatic detection system is presented in Fig. 5. Each block is described in the following subsections:

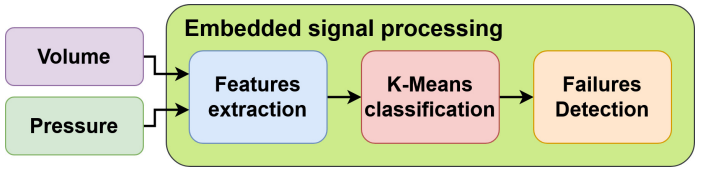

Fig. 5: Hardware failure automatic detection system.

\section{A. Features extraction}

First step of the proposed algorithm is to extract relevant features from sensors signals on each breath cycle as shown Fig. 6. Following characteristics features are extracted and used as inputs of the unsupervised learning algorithm:

- Maximum intra-lung pressure $\left(P_{p}\right)$ in millibar.

- Expired volume $\left(P_{v}\right)$ in litres.

- Area under the pressure curve $\left(A_{p}\right)$.

- Area under the volume curve $\left(A_{v}\right)$.

- Pressure pulse duration $\left(D_{p}\right)$ in seconds.

- Volume pulse duration $\left(D_{v}\right)$ in seconds.

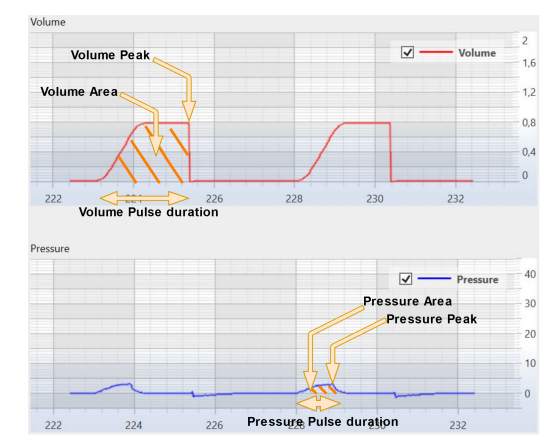

Fig. 6: Volume and pressure features extraction.

\section{B. K-Means unsupervised learning and detection algorithm}

Considering that the ventilator will be used with different patients and settings, learning use case reference situation is necessary for later detecting issues. This is done in unsupervised learning using a K-Means optimized algorithm, classifying features in real time without storing any dataset [10]-[12]. This allows an embedded implementation in a microcontroller, improving system reliability. Training and detection algorithms are presented in Figs. $7 \mathrm{a}$ and $7 \mathrm{~b}$.

Learning phase is preceded by an initialisation phase. First $k$ samples are used for evaluating data dispersion by calculating the standard deviation for each feature $\left(\bar{\sigma}=\left[\overline{\sigma_{P_{p}}}, \overline{\sigma_{P_{v}}}, \overline{\sigma_{A_{p}}}, \overline{\sigma_{A_{v}}}, \overline{\sigma_{D_{p}}}, \overline{\sigma_{D_{v}}}\right]\right)$.

Then in training phase, sets of features (composed by 6 elements having been described in Section VI-A) are assigned to existing clusters, if they are close to an existing one, or to a new one if not. A cluster $C^{j}$ (where $j$ is the cluster ID), represents a behaviour of the couple $\{$ ventilator-patient $\}$. A cluster is described by its centroid $C^{j}=\left[\overline{P_{p_{j}}}, \overline{P_{v_{j}}}, \overline{A_{p_{j}}}, \overline{A_{v_{j}}}, \overline{D_{p_{j}}}, \overline{D_{v_{j}}}\right]$ and its dispersion $\sigma_{C^{j}}=\left[\sigma_{P_{p_{j}}}, \sigma_{P_{v_{j}}}, \sigma_{A_{p_{j}}}, \sigma_{A_{v_{j}}}, \sigma_{D_{p_{j}}}, \sigma_{D_{v_{j}}}\right]$. Notations $C^{j}=$ $\left[C_{1}^{j}, \ldots, C_{6}^{j}\right]$ and $\sigma_{C^{j}}=\left[\sigma_{C_{1}^{j}}, \ldots, \sigma_{C_{6}^{j}}\right]$ are used for simplifying equations. $n_{j}$ is the number of samples classified in cluster $j$. Each time a feature has been extracted, distance $D$ between its signature $S=\left[P_{p}, P_{v}, A_{p}, A_{v}, D_{p}, D_{v}\right]$ and each cluster is evaluated using Eq. 2 .

$$
D=\sqrt{\sum_{i=1}^{6}\left(\frac{S_{i}-C_{i}^{j}}{\sigma_{C_{i}^{j}}}\right)^{2}}
$$

Distance $D$ is compared to a threshold $D_{\operatorname{Max}}$ optimized offline using deep learning. Its value is equal to 1.25 . This optimization is not the purpose of this paper.

If $D \leq D_{\text {Max }}$, sample is assigned to cluster $j$. Consequently as the sample is assigned to cluster $j$, each component of its centroid and their corresponding dispersions are updated as shown in Eq. $3 \mathrm{a}$ and Eq. $3 \mathrm{~b}$ :

$$
\begin{aligned}
C_{i}^{j} & =(1-\alpha) C_{i}^{j}+\alpha S_{i} \\
\sigma_{C_{i}^{j}} & =(1-\alpha) \sigma_{C_{i}^{j}}+\alpha\left|S_{i}-C_{i}^{j}\right| \\
n_{i} & =n_{i}+1
\end{aligned}
$$

Eq. $3 \mathrm{a}$ and $3 \mathrm{~b}$ shows that $C_{i}^{j}$ and $\sigma_{C_{i}^{j}}$ are moving averages of $S_{i}$ and $\left|S_{i}-C_{i}^{j}\right|$, implemented as low pass filters. This implementation is much lighter than using an arithmetical mean and adapts to slow changes in cluster parameters. Number of elements of the cluster $n_{i}$, incremented in Eq. 3c gives indications on cluster usefulness. Only most important ones will be kept at the end of learning phase.

If $D>D_{\text {Max }}$, there are two options:

- If algorithm is in learning phase, a new cluster $C^{j}$ is created as shown Fig. 7a its centroid parameters are initialized with the new sample signature and its disper- 
sion is initialized considering the overall data dispersion calculated in the initialisation phase, as shown in Eq. 4

$$
C_{i}^{j}=S_{i} \quad \sigma_{C_{i}^{j}}=\frac{\overline{\sigma_{i}}}{2}
$$

- If algorithm is in detection phase, an alert is generated as shown in Fig. $7 \mathrm{~b}$ Fig. 8 shows a screen-shot of the interface during monitoring, two clusters can be observed represented in 3D with the most relevant features.
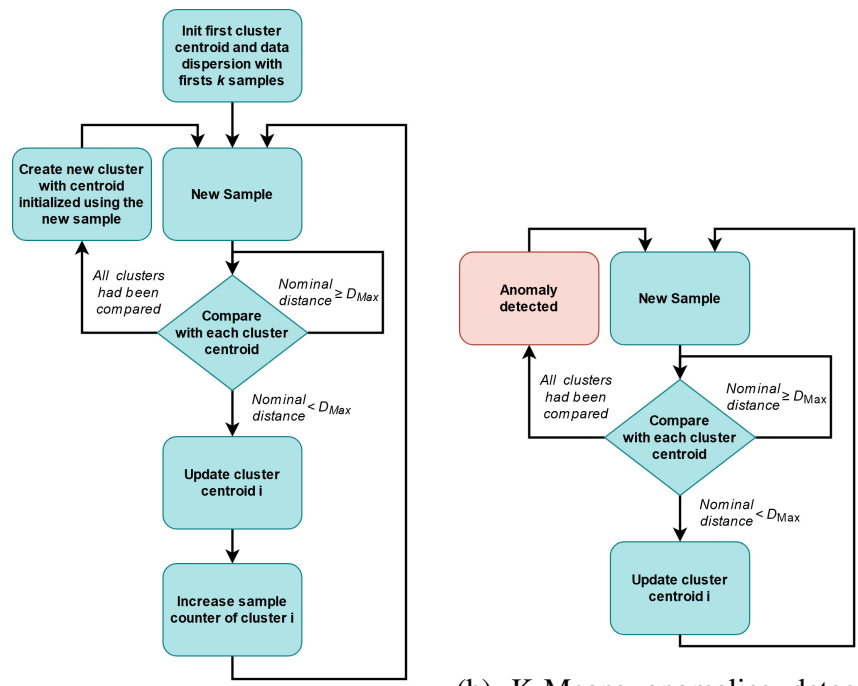

(a) K-Means training algorithm.

(b) K-Means anomalies detection algorithm.

Fig. 7: Unsupervised learning algorithms.

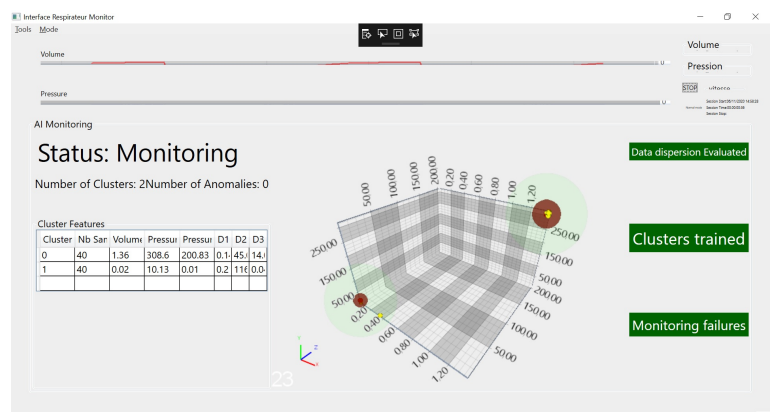

Fig. 8: Interface during monitoring phase

\section{RESULTS}

Proposed artificial ventilator has been successfully tested at IRBA (Institute de Recherche Biomédical des Armées) using a reference artificial lung. For evaluating the performance of the fault detector, a learning phase without failures has been done. Then, several tests were performed simulating mechanical issues after learning the baseline condition: disconnection or closure of pipes, structure breaking, fishing wires cut as well as cut of fellows elastic strap. Every failure was detected except in one situation: when a fishing wire is cut on only one of three air bellows, if maximal intra-lung pressure is limited to 15 mbar and volume required is lower than $0.9 \mathrm{~L}$, no alarm is generated because normal operation can be performed using only 2 air bellows instead of 3 . Fault detection sensitivity can be tuned by adjusting cluster belonging tolerance $D_{\text {Max }}$.

\section{CONCLUSION}

In this paper, a less than $\$ 200$ artificial ventilator that can help COVID-19 patients has been proposed. Using lowcost easy-to-find materials, it has been designed for helping developing countries where ventilators and medical supplies are hard to find. Despite its very low cost, it is compliant with most important medical requirements and has been tested by IRBA, the French Biomedical Defence Institute. Most parameters such as inspired volume, maximum intra-lung pressure and breath rate can be adjusted and monitored.

Moreover, considering that it is a life equipment built with low-cost easy-to-find and recycled materials having an unknown quality, an intelligent embedded hardware failure detector has been implemented and embedded in the microcontroller. In case of abnormal functioning an alarm is generated to warn the medical staff.

Its mechanical, electrical and software architectures are open source and published in [9] and on GitHub: https://github.com/iutgeiitoulon/ArtificialVentilator

\section{ACKNOWLEDGEMENT}

The authors would like to thank the Embedded Electronics Technology Platform of Toulon University named SMIoT [9] for its support in the achievement of this work.

\section{REFERENCES}

[1] S. C. Saxena, P. Mukhopadhyay, and H. K. Verma, "Respirator design," IEE-IERE Proceedings - India, vol. 12, no. 6, pp. 235-239, 1974.

[2] Y. Mitamura, T. Mikami, H. Sugawara, and C. Yoshimoto, "An optimally controlled respirator," IEEE Transactions on Biomedical Engineering, vol. BME-18, no. 5, pp. 330-338, 1971.

[3] M. Stork and J. Ruzicka, "Artificial lung ventilator working in the hyperbaric chamber," in 2006 International Conference on Applied Electronics, 2006, pp. 189-192.

[4] M. Shahid, "Prototyping of artificial respiration machine using ambu bag compression," in 2019 International Conference on Electronics, Information, and Communication (ICEIC), 2019, pp. 1-6.

[5] H. Langenstein, "[emergency ventilation using an ambu-bag with a new coniotomy set]," Der Anaesthesist, vol. 35, no. 11, p. 697-699, November 1986. [Online]. Available: http://europepmc.org/abstract/ MED/3812967

[6] M. R. Islam, M. Ahmad, M. S. Hossain, M. Muinul Islam, and S. F. Uddin Ahmed, "Designing an electro-mechanical ventilator based on double cam integration mechanism," in 2019 1st International Conference on Advances in Science, Engineering and Robotics Technology (ICASERT), 2019, pp. 1-6.

[7] J. Capelli, N. Ferlo, A. Foley, V. Smith, and G. Atlas, "A novel system to assist in manual resuscitation and detect spontaneous breathing," in 2011 IEEE 37th Annual Northeast Bioengineering Conference (NEBEC), 2011, pp. 1-2.

[8] C. D. Lellis and L. Székelyhidi, "The euler equations as a differential inclusion," Annals of Mathematics, vol. 170, no. 3, pp. 1417-1436, 2009. [Online]. Available: http://www.jstor.org/stable/25662181

[9] V. Gies, V. Barchasz, and H. Glotin, "Smiot: Scientific microsystems for the internet of things, technological platform," http://www.smiot.fr, La Garde, France. [Online]. Available: http://www.smiot.fr

[10] S. Khanmohammadi, N. Adibeig, and S. Shanehbandy, "An improved overlapping k-means clustering method for medical applications," Expert Systems with Applications, vol. 67, pp. 12-18, 2017.

[11] T. Chen, C. Sun, H. Su, S. Chien, D. Deguchi, I. Ide, and H. Murase, "Power-efficient hardware architecture of k-means clustering with bayesian-information-criterion processor for multimedia processing applications," IEEE Journal on Emerging and Selected Topics in Circuits and Systems, vol. 1, no. 3, pp. 357-368, 2011

[12] T. Kanungo, D. M. Mount, N. S. Netanyahu, C. D. Piatko, R. Silverman, and A. Y. Wu, "An efficient k-means clustering algorithm: analysis and implementation," IEEE Transactions on Pattern Analysis and Machine Intelligence, vol. 24, no. 7, pp. 881-892, 2002. 